Supporting information for

\title{
Pd Nanoclusters Supported by Amine- Functionalized Covalent Organic Frameworks for Benzyl Alcohol Oxidation
}

Congying Xu, ${ }^{\dagger}$ Junyu Lin, ${ }^{\dagger}$ Dan Yan, ${ }^{\dagger}$ Zhiyong Guo, ${ }^{* \dagger, \S}$ Douglas J. Austin Jr, ${ }^{\perp}$ Hongbing Zhan, ${ }^{\dagger}$ Atavia Kent, ${ }^{\perp}$ and Yanfeng Yue* ${ }^{\perp}$

${ }^{\dagger}$ College of Materials Science and Engineering, Fuzhou University, Fuzhou 350108, Fujian, P. R. China

†Testing Center, Fuzhou University, Fuzhou 350108, Fujian, P. R. China

§ Key Laboratory of Eco-materials Advanced Technology, Fuzhou University, Fuzhou 350108, Fujian, P. R. China*E-mail: guozhy@fzu.edu.cn (Z.G.).

${ }^{\perp}$ Department of Chemistry, Delaware State University, Dover, Delaware 19901, United States

*E-mail: yyue@desu.edu (Y.Y.). 


\section{Experimental and characterizations}

\section{Materials}

All chemical reagents and solvents were purchased from commercial suppliers and used without further purification, among which tetra-(4-anilyl)-methane was obtained from Bide Pharmatech Ltd.

\section{Characterizations}

Powder X-ray diffraction patterns of the samples were obtained by a Rigaku Ultima III X-ray diffractometer using $\mathrm{Cu} \mathrm{K} \alpha$ radiation $(36 \mathrm{kV}, 30 \mathrm{~mA}$ ). The Fourier Transform Infrared (FTIR) spectra was measured on a Thermo Fisher Scientific Nicolet 5700 FT-IR spectrometer. The BET surface area measurements were performed on a Micromeritics ASAP 2020 gas sorption analyser. Before analysis, samples were charged into a sample tube and activated at $100{ }^{\circ} \mathrm{C}$ until pressure reached $5 \mu \mathrm{mHg}$. High purity gases $\left(\mathrm{N}_{2}: 99.9999 \%\right)$ were used for the gas sorption measurements. Gas chromatography (GC) analysis was performed using an Agilent 7890B gas chromatograph equipped with a HP-5 capillary column $(30 \mathrm{~m} \times 0.32 \mathrm{~mm} \times 0.25$ $\mu \mathrm{m})$ and a flame ionization detector. Mesitylene was used as the internal standard. The transmission electron microscopy was recorded on a FEI Tecnai-G2-F20 Transmission electronic microscope operated at $200 \mathrm{kV}$. Pd content in COF-300 was determined using inductively coupled plasma mass spectrometry (ICP-MS). X-ray photoelectron spectroscopy (XPS) was characterized by Thermo Scientific K-Alpha+ spectrometer with a monochromatized Al Ka X-ray source $(\mathrm{h} v=1486.6 \mathrm{eV})$.

Synthesis of COF-300-xy: all of COF-300 samples were synthesized according to the procedure reported in the literature with some modifications on monomer ratio. Typically, a Pyrex tube measuring $12 \times 8 \mathrm{~mm}$ (o.d $\times$ i.d) was charged with tetra-(4-anilyl)-methane ( 66.5 $\mathrm{mg}, 0.175 \mathrm{mmol})$ and terephthaldehyde $(47.25 \mathrm{mg}, 0.35 \mathrm{mmol})$ in a mixed solution of dioxane $(1.5 \mathrm{~mL})$ and $3 \mathrm{M}$ aqueous acetic acid $(0.3 \mathrm{~mL})$. The tube was flash frozen with a liquid N2 bath and degassed for three freeze-pump-thaw cycles. Subsequently, the tube was evacuated and flame sealed. The reaction was heated at $120{ }^{\circ} \mathrm{C}$ for $72 \mathrm{hrs}$ yielding yellowish solids, described as COF-300-xy, where $\mathrm{x}$ and $\mathrm{y}$ are numerator and denominator of the ratio for TPA and TAM, respectively. For instance, here COF-300-0714 means the molar ratio of terephthaldehyde to tetra-(4-anilyl)-methane is $07 / 14(0.175 \mathrm{mmol}$ to $0.35 \mathrm{mmol})$. The resultant powder was isolated by filtration and washed with dioxane and tetrahydrofuran (THF). Finally, the product was extracted with THF in a Soxhlet apparatus for $24 \mathrm{hrs}$. By using this synthesis approach, the yields for the preparation COF-300-xy series almost are same (around $62 \%$ based on the input of TPA).

The preparation of Pd/COF-300-xy: $80 \mathrm{mg}$ of dried COF-300-xy was dispersed in dichloromethane $(8 \mathrm{~mL})$. A dichloromethane solution $(2 \mathrm{~mL})$ containing $8.84 \mathrm{mg} \mathrm{Pd}(\mathrm{OAc})_{2}$ was added dropwise to the above solution under vigorous magnetic stirring. The mixture was 
stirred at room temperature for $24 \mathrm{hrs}$. The sample was isolated and washed with dichloromethane five times by centrifugation and then dried under ambient condition. Absorbed $\mathrm{Pd}$ (II) ions in COF-300-xy samples were further reduced in a $10 \% \mathrm{H}_{2} / \mathrm{Ar}$ flow $\left(\mathrm{H}_{2} / \mathrm{Ar}=5 / 45 \mathrm{~mL} / \mathrm{min}\right)$ at $200{ }^{\circ} \mathrm{C}$ for $2 \mathrm{hrs}$ resulting in $\mathrm{Pd} / \mathrm{COF}-300-x y$. The Pd content of the products was determined based on the ICP measurements.

\section{Heterogeneous catalytic benzyl alcohol oxidation}

A $10 \mathrm{~mL}$ Shlenk flask fitted with a condenser and a magnetic stirrer was charged with $10 \mathrm{mg}$ catalyst, $0.2 \mathrm{mmol}$ benzyl alcohol, and $3 \mathrm{~mL}$ toluene. The flask was then saturated with $\mathrm{O}_{2}$ and sonicated for $10 \mathrm{~min}$. Subsequently, the mixture was heated up to $90{ }^{\circ} \mathrm{C}$ under stirring at atmospheric $\mathrm{O}_{2}$ pressure. After $24 \mathrm{hrs}$, the reaction mixture was immediately separated by centrifugation. The products were analyzed on a HP 7890B gas chromatograph equipped with a HP-5 capillary column. The recovered catalyst was washed with $\mathrm{MeOH}$ for several times and further evacuated at $60{ }^{\circ} \mathrm{C}$ for $12 \mathrm{hrs}$. The recovered catalyst was reused for the successive catalytic run. Though, the Pd NPs may be partially oxidized after the reaction. However, it is difficult to draw firm conclusion that the amine groups that protected NCs were oxidized after reaction.

\section{Leaching experiments}

After reaction, the filtrate collected by centrifugation was transferred to another glass reactor under the same conditions and the reaction was continued for an additional $60 \mathrm{hrs}$ without a catalyst. GC analysis was conducted every $12 \mathrm{hrs}$. 


\section{Nitrogen isotherms of COF-300-xy series.}

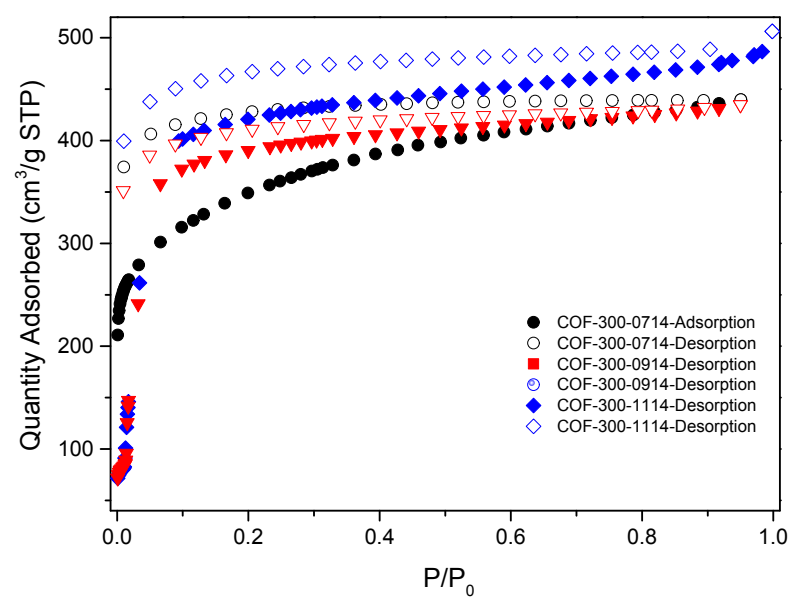

Figure S1. The comparasion of nitrogen adsorptions of COF-300- $x y$ ( $x y=0714,0914$, and 1114 ) series at $77 \mathrm{~K}$. For all these three isoterms, the desorption ranch not matches with related adsorption branch, resulting in a big hyteresis in whole range of pressure. These phenominia are quite normal for COFs, particularly for $2 \mathrm{D}$ COFs. ${ }^{1-3}$

1. Y. Li, W. Chen, R. Gao, Z. Zhao, T. Zhang, G. Xing, L. Chen, Chem. Commun. 2019, 55, 14538-14541.

2. Y. Chen, Z.-L. Shi, L. Wei, B. Zhou, J. Tan, H.-L. Zhou, Y.-B. Zhang, J. Am. Chem. Soc. 2019, 141, 3298-3303.

3. F. J. Uribe-Romo, J. R. Hunt, H. Furukawa, C. Klöck, M. O’Keeffe, O. M. Yaghi. J. Am. Chem. Soc. 2009, 131, 4570-4571. 
2. Porosity summary of COF-300-xy $(x y=0714,0914$, and 1114) series (Table S1)

\begin{tabular}{|c|c|c|c|c|c|c|c|}
\hline COF-300- $x y$ & $\begin{array}{c}\mathrm{S}_{\text {BET }^{\mathrm{a}}} \\
\left(\mathrm{m}^{2} \mathrm{~g}^{-1}\right)\end{array}$ & $\begin{array}{c}\mathrm{S}_{\text {micro }} \mathrm{b} \\
\left(\mathrm{m}^{2} \mathrm{~g}^{-1}\right)\end{array}$ & $\mathrm{S}_{\text {micro }} / \mathrm{S}_{\mathrm{BET}}$ & $\begin{array}{c}\mathrm{V}_{\text {total }}^{\mathrm{c}} \\
\left(\mathrm{cm}^{3} \mathrm{~g}^{-1}\right)\end{array}$ & $\begin{array}{c}\mathrm{V}_{\text {micro }}{ }^{\mathrm{d}} \\
\left(\mathrm{cm}^{3} \mathrm{~g}^{-1}\right)\end{array}$ & $\mathrm{V}_{\text {micro }} / \mathrm{V}_{\text {total }}$ & $\begin{array}{c}\mathrm{D}_{\text {pore }}{ }^{\mathrm{e}} \\
(\mathrm{nm})\end{array}$ \\
\hline COF-300-0714 & 1188 & 1137.7 & 0.96 & 0.68 & 0.60 & 0.88 & $1.9,2.3,2.6,3.1$ \\
\hline COF-300-0914 & 1233 & 1181.1 & 0.96 & 0.62 & 0.56 & 0.90 & 1.9 \\
\hline COF-300-1114 & 1299 & 1139.4 & 0.88 & 0.74 & 0.60 & 0.81 & 1.9 \\
\hline
\end{tabular}

${ }^{a}$ BET specific surface area calculated at the relative pressure ranging from 0.01 to 0.23 . ${ }^{b}$ Micropore surface area using the $t$-plot method. ${ }^{c}$ Total pore volume at $P / P_{0}=0.95 .{ }^{d}$ Micropore volume calculated using the $t$-plot method. ${ }^{e}$ Dominant pore size determined by NLDFT model (option: $\mathrm{N}_{2}$ at $77 \mathrm{~K}$ on carbon, slit pore). 
3. The PSDs of COF-300- $x y$ series
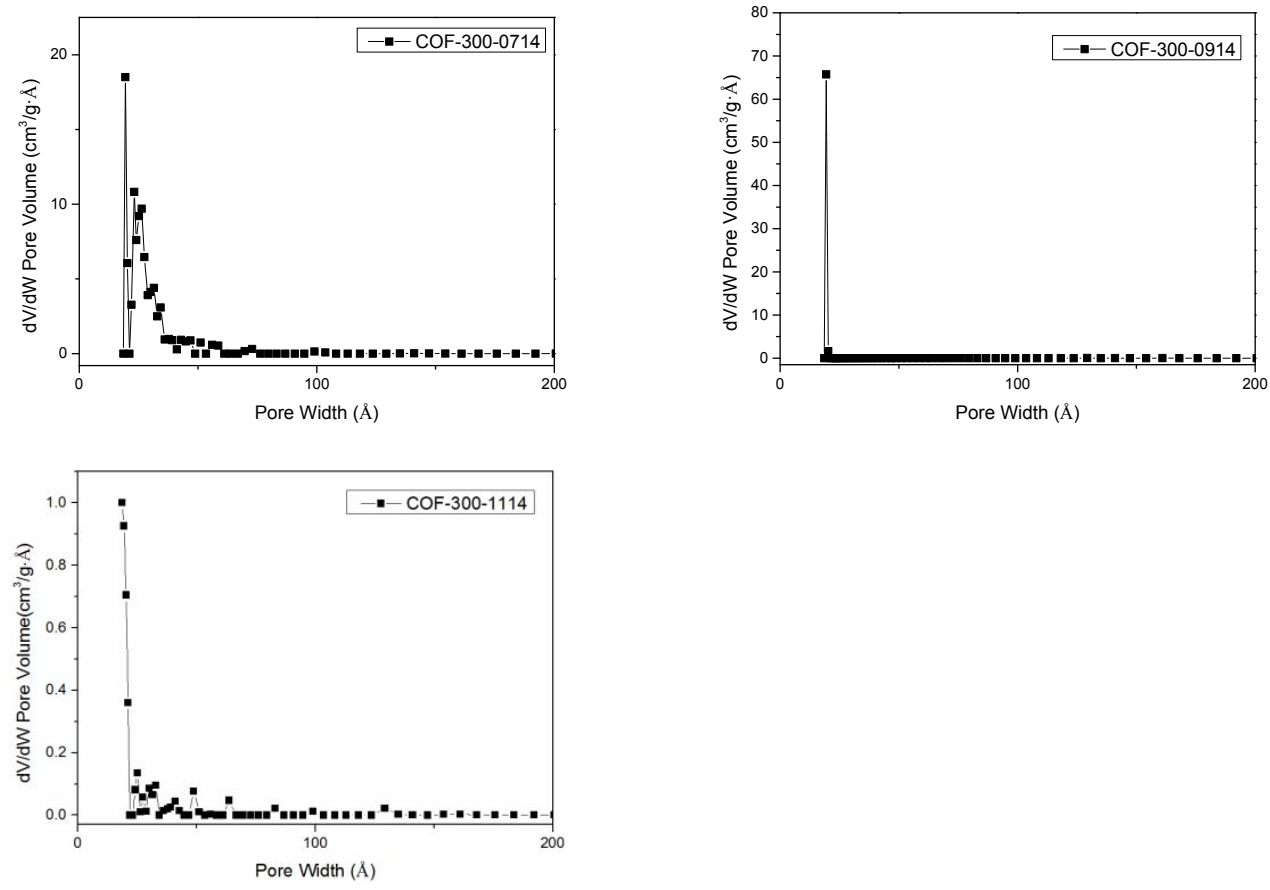

Figure S2. The PSDs of COF-300- $x y$ ( $x y=0714,0914$, and 1114) series, calculated from related adsorption branch of the nitrogen isotherms collected at $77 \mathrm{~K}$. 


\section{Comparison of FT-IR spectra of Pd/COF-300-xy}

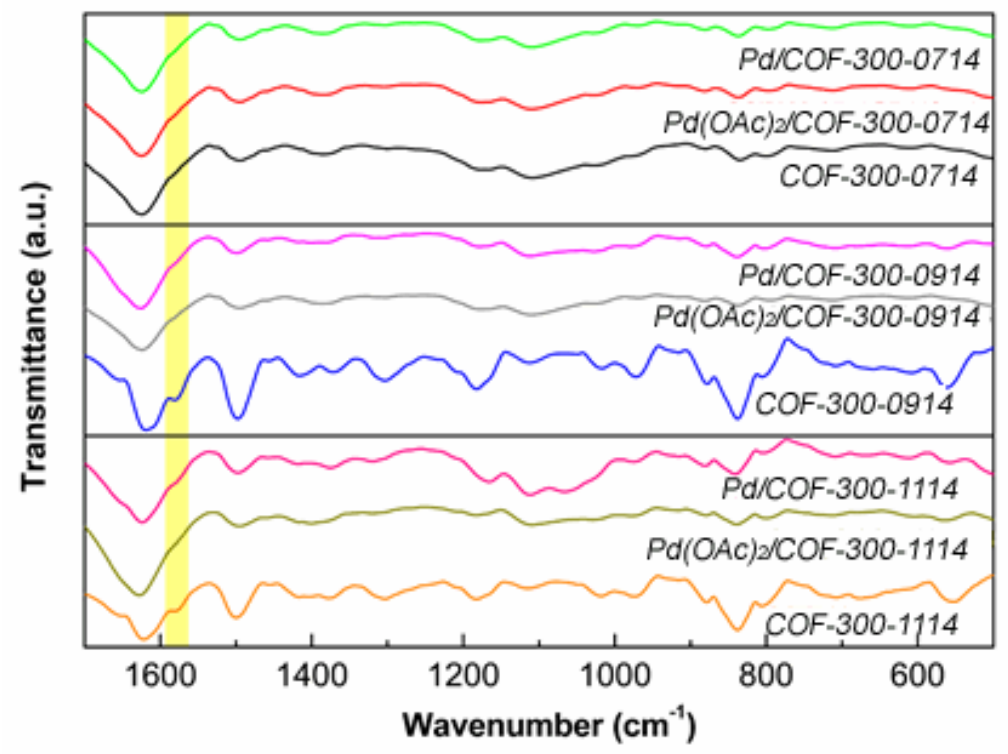

Figure S3. The comparasion of FT-IR spectra of Pd/COF-300-xy, $\mathrm{Pd}(\mathrm{OAc})_{2} / \mathrm{COF}-300-x y$, and COF-300-xy $(x y=0714,0914$, and 1114). As shown in FT-IR spectra, the peak attributed to $\mathrm{N}-\mathrm{H}$ bending vibration appeared at $1580 \mathrm{~cm}^{-1}$, which was only observed in COF-300-0914 and COF-300-1114, indicating the existence of excess $\mathrm{NH}_{2}$ groups in these frameworks. This peak was found to be weaker after solution impregnation of $\mathrm{Pd}(\mathrm{OAc})_{2}$ and $\mathrm{H}_{2}$ reduction. XRD and FT-IR analysis for Pd/COF-300-xy showed no qualitative difference compared to the parent COF-300-xy, demonstrating that the host framework integrity was still maintained after the introduction of the palladium into COF-300 structure. Besides, no X-ray diffraction peaks from Pd nanocrystal were observed in PXRD patterns, suggesting that the size of encapsulated Pd particles were very small. 
5. The XRD patterns of Pd/COF-300-1114

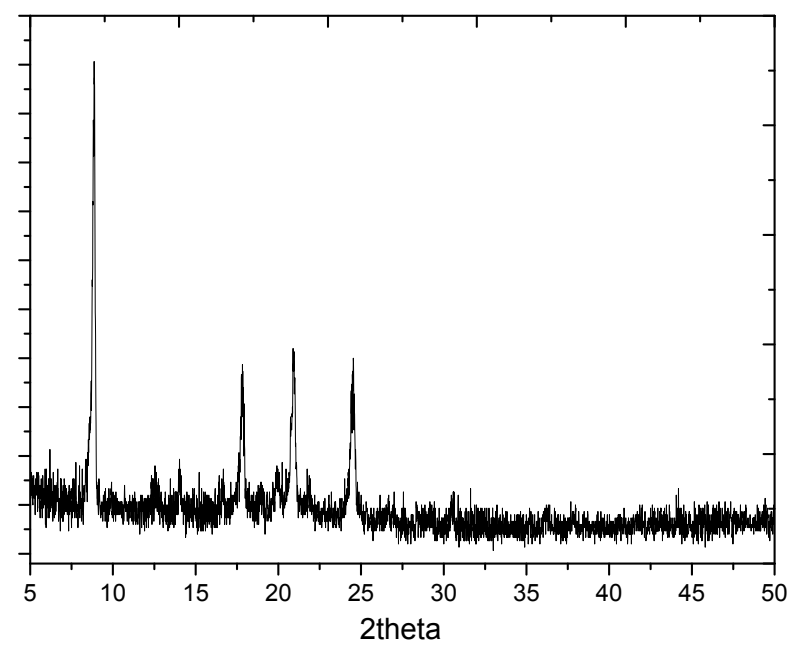

Figure S4. The XRD patterns of Pd/COF-300-1114 (detection angle 5-50').

S8 
6. Mapping and EDX images for Pd/COF-300-1114

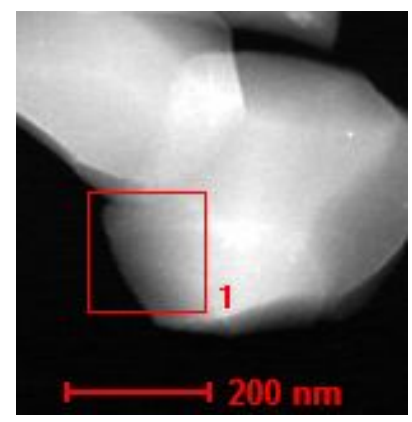

Figure S5. The STEM images of Pd/COF-300-1114.
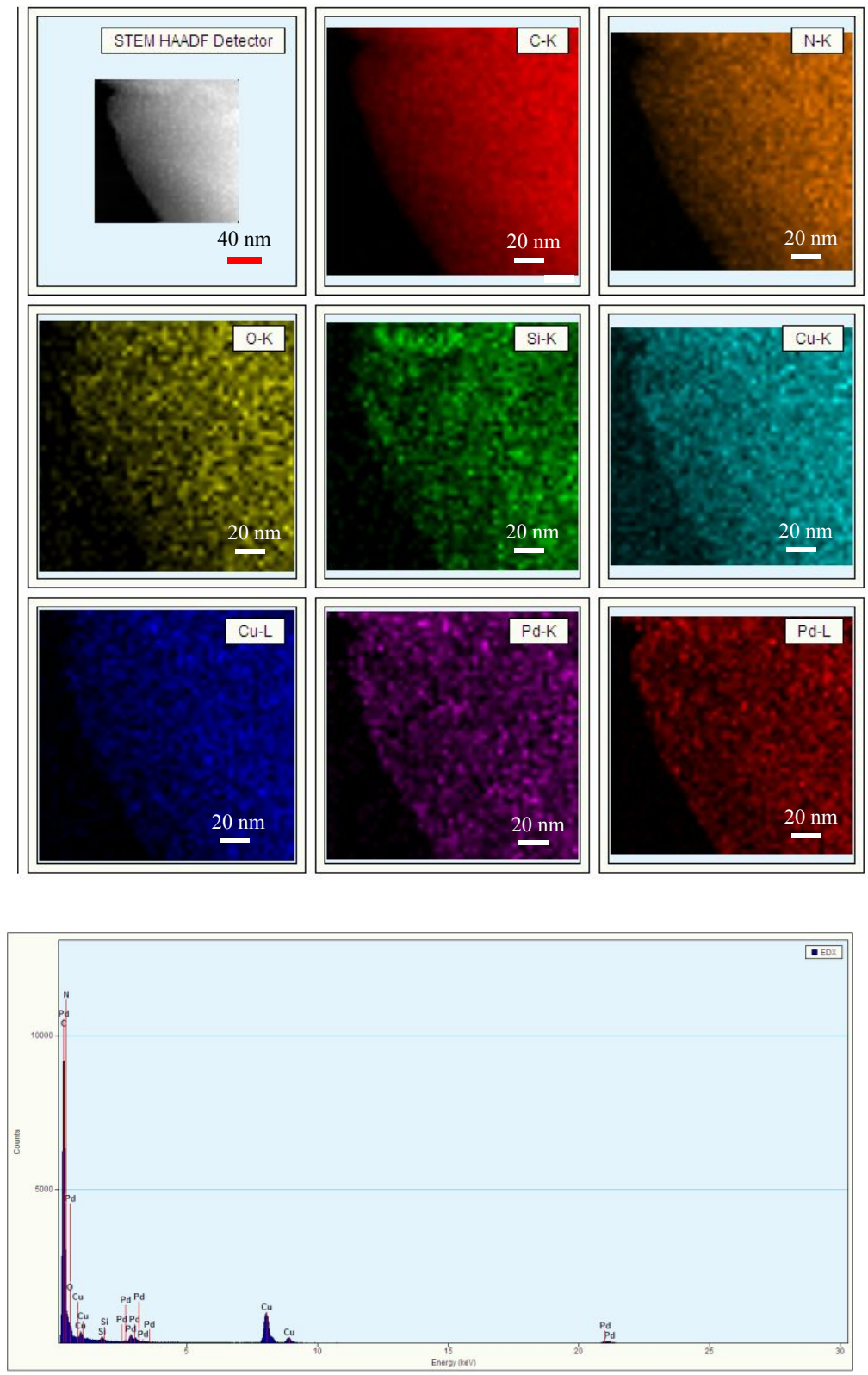

Figure S6. The elements present in Pd/COF-300-1114 were confirmed by the SEM-energydispersive X-ray (EDX). The EDX mapping also showed a homogeneous distribution of $\mathrm{N}, \mathrm{O}$, and Pd elements in this material. 
7. ICP-MS result of Pd content in COF-300- $x y(x y=0714,0914$, and 1114) (Table S2)

\begin{tabular}{|l|c|c|c|}
\hline & Pd/COF-300-0714 & Pd/COF-300-0914 & Pd/COF-300-1114 \\
\hline Pd wt $\%$ & $2.0 \%$ & $1.3 \%$ & $1.1 \%$ \\
\hline Pd loading efficency & $41 \%$ & $26 \%$ & $22 \%$ \\
\hline
\end{tabular}



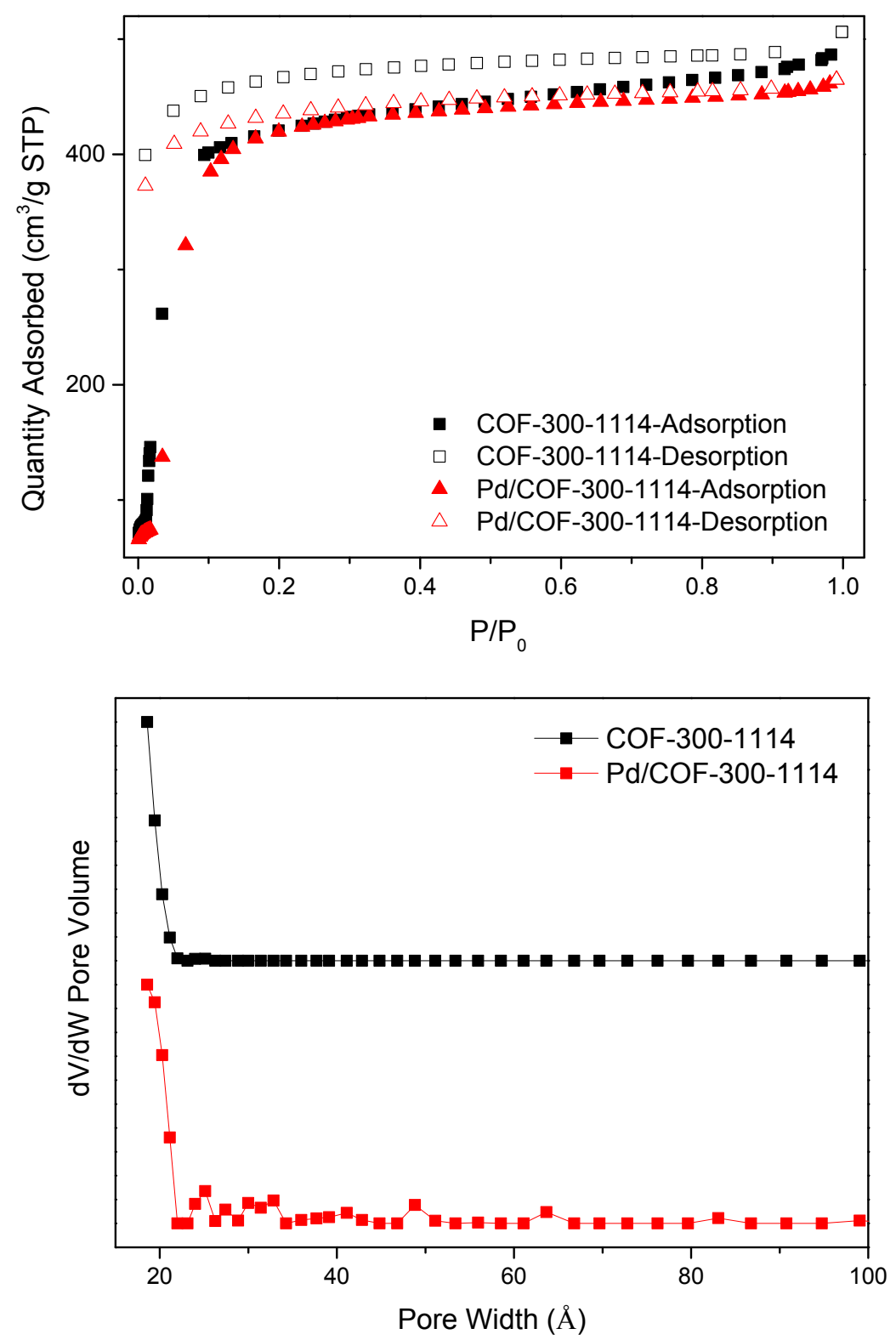

Figure S7. The nitrogen isotherms of COF-300-1114 and Pd/COF-300-1114 at $77 \mathrm{~K}$. All adsorption-desorption isotherms show a type I shape, implying the COFs' microporous nature. The BET surface area and pore volume of COF-300-1114 were $1299 \mathrm{~m}^{2} \mathrm{~g}^{-1}$ and 0.74 $\mathrm{cm}^{3} \mathrm{~g}^{-1}$, respectively. Compared to the parent COF-300 frameworks, the BET surface area and pore volume of $\mathrm{Pd} / \mathrm{COF}-300-1114$ decreased to $1264 \mathrm{~m}^{2} \mathrm{~g}^{-1}$ and $0.66 \mathrm{~cm}^{3} \mathrm{~g}^{-1}$, respectively, mainly attributed to the occupation of the cages of COF-300-1114 by the Pd NCs. 


\section{Porosity sumamry of COF-300-1114 and Pd/COF-300-1114 (Table S3)}

\begin{tabular}{|c|c|c|c|c|c|c|c|}
\hline $\mathrm{COF}$ & $\begin{array}{l}\mathrm{S}_{\mathrm{BET}^{a}} \\
\left(\mathrm{~m}^{2} \mathrm{~g}^{-1}\right)\end{array}$ & $\begin{array}{l}\mathrm{S}_{\text {micro }}{ }^{b} \\
\left(\mathrm{~m}^{2} \mathrm{~g}^{-1}\right)\end{array}$ & $\mathrm{S}_{\text {micro }} / \mathrm{S}_{\mathrm{BET}}$ & $\begin{array}{l}\mathrm{V}_{\text {total }}{ }^{\mathrm{c}} \\
\left(\mathrm{cm}^{3} \mathrm{~g}^{-1}\right)\end{array}$ & $\begin{array}{l}\mathrm{V}_{\text {micro }}{ }^{d} \\
\left(\mathrm{~cm}^{3} \mathrm{~g}^{-1}\right)\end{array}$ & $\mathrm{V}_{\text {micro }} / \mathrm{V}_{\text {total }}$ & $\begin{array}{l}\mathrm{D}_{\text {pore }}{ }^{e} \\
(\mathrm{~nm})\end{array}$ \\
\hline & 1299 & 1139 & 0.8 & 0.74 & 0.60 & 0.81 & 1.9 \\
\hline $\mathrm{Pd} / \mathrm{COF}-300-1114$ & 1264 & 1207 & 0.94 & 0.66 & 0.58 & 0.87 & 1.9 \\
\hline
\end{tabular}

${ }^{a}$ BET specific surface area calculated at the relative pressure ranging from 0.01 to 0.23 . ${ }^{b}$ Micropore surface area using the $t$-plot method. ${ }^{c}$ Total pore volume at $P / P_{0}=0.95$. ${ }^{d}$ Micropore volume calculated using the $t$-plot method. ${ }^{e}$ Dominant pore size determined by NLDFT model (option: $\mathrm{N}_{2}$ at $77 \mathrm{~K}$ on carbon, slit pore). 
10. XPS spectra for Pd/COF-300-xy
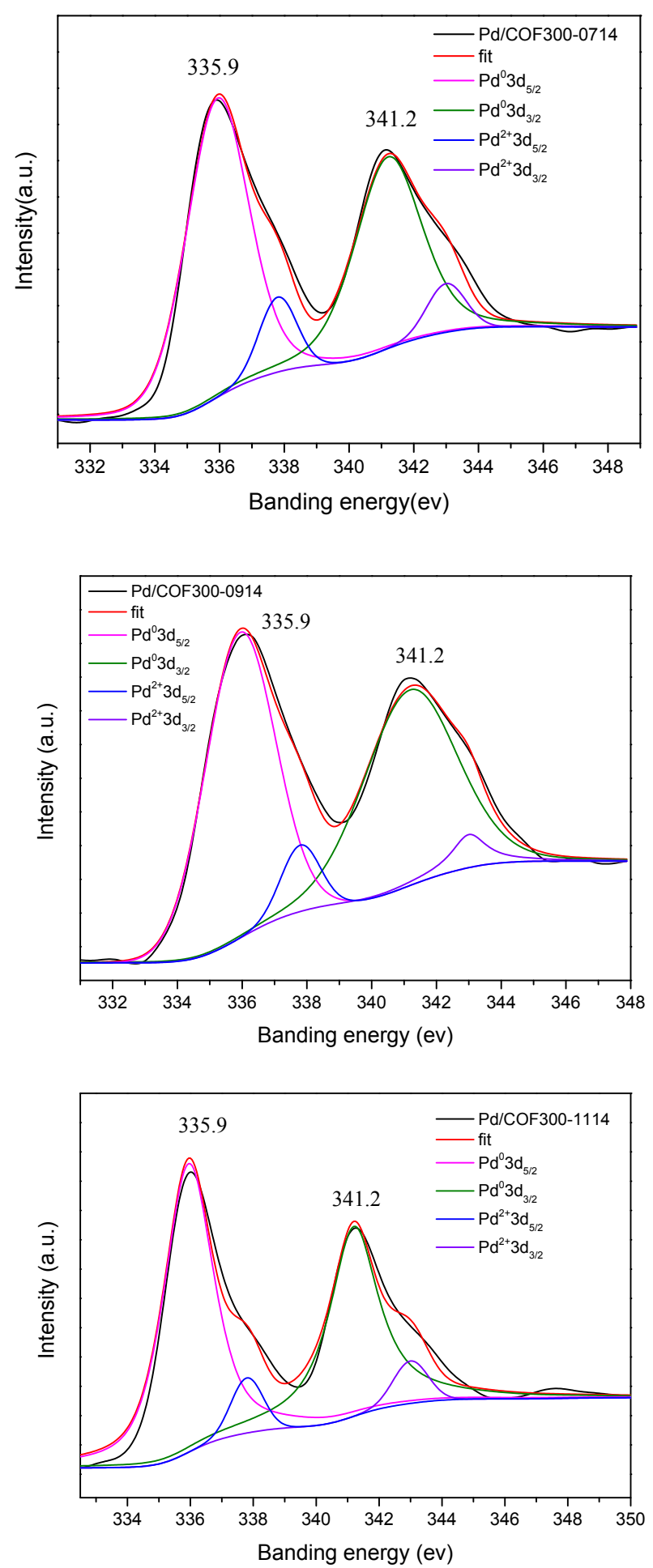

Figure S8. The XPS measurements of Pd/COF-300-0714 (top), Pd/COF-300-0914 (middle), and $\mathrm{Pd} / \mathrm{COF}-300-1114$ (bottom). The peaks at 335.9 and $341.2 \mathrm{eV}$ are characteristic $3 \mathrm{~d}_{5 / 2}$ and $3 d_{3 / 2}$ states of $\operatorname{Pd}(0)$, demonstrated the successful reduction from $\operatorname{Pd}(\mathrm{II})$ to $\operatorname{Pd}(0)$. Whereas the small peaks deconvoluted at 337.8 and $343.0 \mathrm{eV}$ are from $\mathrm{Pd}^{2+} 3 \mathrm{~d}_{5 / 2}$ and $3 \mathrm{~d}_{3 / 2}$ states of $\mathrm{PdO}$, which is from the oxidation of Pd NCs. 


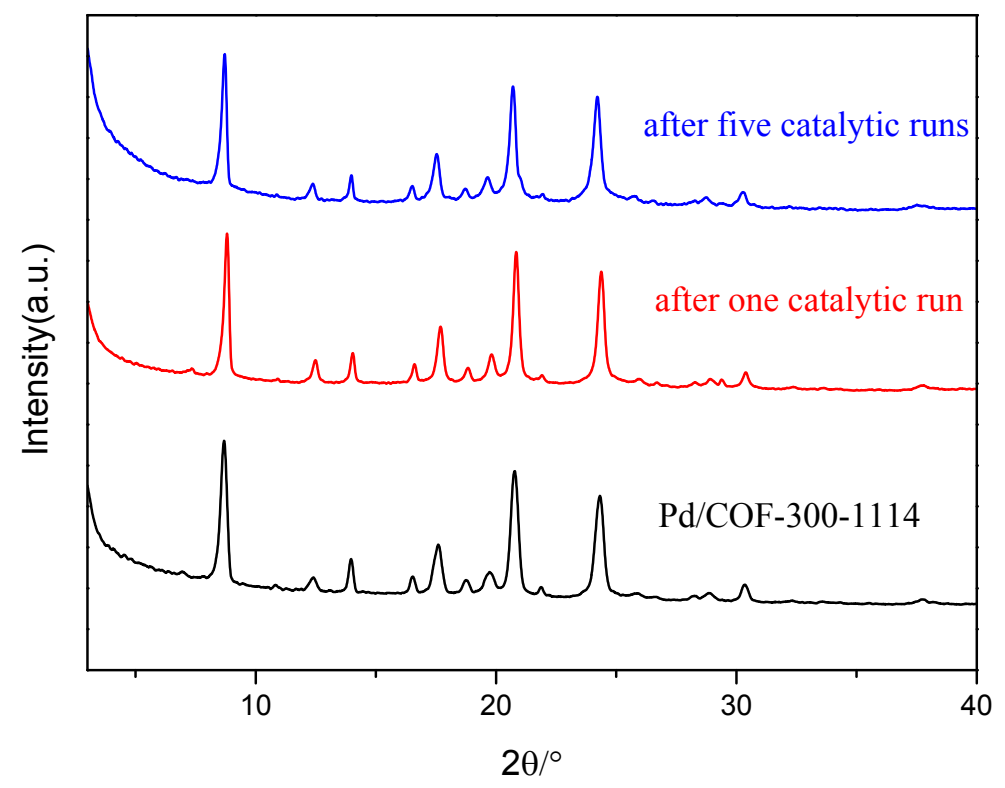

Figure S9. Powder XRD patterns of $\mathrm{Pd} / \mathrm{COF}-300-1114$ after the catalytic reactions. A comparison of the powder XRD patterns of used Pd/COF-300-1114 and Pd/COF-300-1114, which revealed that the $\mathrm{Pd} / \mathrm{COF}$ coposite catalyst survived after five time cyclings. 
12. The TEM images of Pd/COF-300-1114 after five times cycling
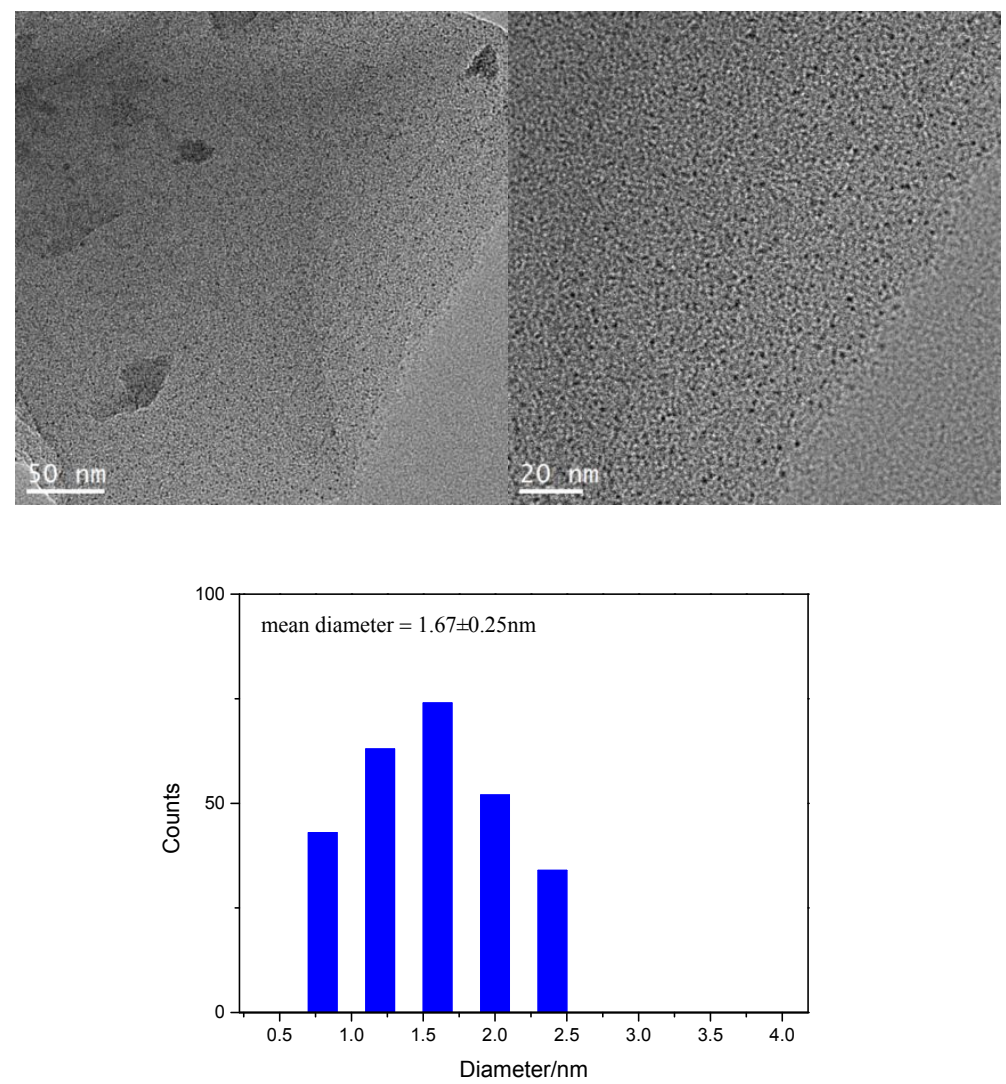

Figure S10. The TEM images with different magnitudes and Pd particle size distribution for the sample of $\mathrm{Pd} / \mathrm{COF}-300-1114$ after five times of cycling for catalytic reaction. 
13. Catalytic performance - leaching test

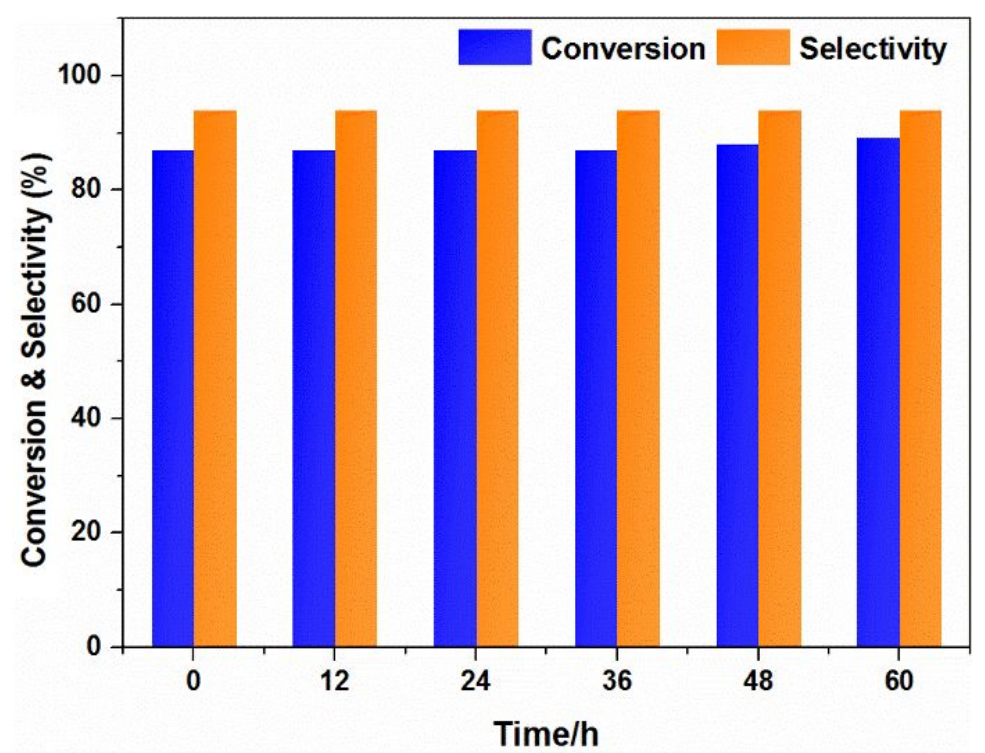

Figure S11. Leaching test for Pd/COF-300-1114. No further conversion of benzyl alcohol was detected during the leaching test experiment, which confirmed the heterogeneity of the catalyst. 